\title{
WHEN DOES RESTRICTED CONTINUITY ON CONTINUOUS FUNCTION GRAPHS IMPLY JOINT CONTINUITY?
}

\author{
JOHN P. DALBEC
}

(Communicated by Andrew M. Bruckner)

\begin{abstract}
We generalize a result of Luzin about real functions of real variables - that $F(x, y)$ is jointly continuous if and only if $F(x, f(x))$ is continuous for every continuous $f$-to functions among certain classes of topological spaces.
\end{abstract}

In [3, pp. 173-176] Luzin states and proves that a function $F:[a, b] \times$ $[c, d] \rightarrow \mathbf{R}$ is continuous if and only if its restriction to the graph of each continuous function $f:[a, b] \rightarrow[c, d]$ and $g:[c, d] \rightarrow[a, b]$ is continuous. In this theorem, necessity is easy to show even when $[a, b],[c, d]$, and $\mathbf{R}$ are replaced by arbitrary topological spaces, so we will concern ourselves with proving sufficiency. An examination of Luzin's sufficiency proof shows that he uses only the hypothesis that $F$ is continuous when restricted to the graph of each continuous function $f:[a, b] \rightarrow[c, d]$, or equivalently that the function $F_{f}:[a, b] \rightarrow \mathbf{R}$ is continuous for each such $f$, where $F_{f}(x)=F(x, f(x))$. Our generalizations will use a form of this hypothesis (Theorem 2) or a slightly stronger one (Theorem 1).

We recall some definitions and results from [1] for easy reference.

A space is called Fréchet if the closure of a set includes precisely the limits of all sequences in the set. A space is called sequential if a set is closed precisely when it includes the limits of all sequences included in the set. We will use many other topological terms defined in $[1,4]$ without explicit mention.

Lemma 1 [1, p. 78]. If a sequence converges to a point, all of its subsequences also converge to that point.

Lemma 2 [1, p. 111]. The product of two first countable spaces is first countable.

Lemma 3 [1, p. 78]. A first countable space is Fréchet and a Fréchet space is sequential.

Lemma 4 [1, p. 271]. The product of two sequential spaces, one of which is sequentially compact, is sequential.

Received by the editors April 8, 1991 and, in revised form, October 16, 1991.

1991 Mathematics Subject Classification. Primary 54C05, 54B10; Secondary 26B05.

The author wishes to thank the referee for many helpful suggestions. This paper is based on the author's Master's thesis at Youngstown State University. 
Our first original lemma will be used to construct a subsequence with unique $x$-coordinates so that a single-valued function can be constructed through the subsequence.

Lemma 5. Let $X$ be a $T_{1}$ space and suppose that the sequence of (not necessarily distinct) points $\left\{x_{n}\right\}$ converges to $x_{0}$, and $x_{n} \neq x_{0}$ for all $n$. Then $\left\{x_{n}\right\}$ has an infinite subsequence of distinct points $\left\{x_{n}^{\prime}\right\}$, which converges to $x_{0}$.

Proof. Left to the reader.

Lemma 6. Let $X$ be Hausdorff, and suppose that the sequence of distinct points $\left\{x_{n}\right\} \subset X$ converges to $x_{0}$ and that $x_{n} \neq x_{0}$ for all $n$. Then there exist pairwise disjoint open neighborhoods $W_{n}$ of $x_{n}$ for all $n>0$.

Proof. Since $X$ is Hausdorff, there exist disjoint open neighborhoods $U_{i j}$ and $U_{j i}$ of $x_{i}$ and $x_{j}$, respectively, for all $i$ and $j$ such that $0 \leq i<j$. Since $\left\{x_{n}\right\}$ converges to $x_{0}$ and $U_{0 j}$ is an open neighborhood of $x_{0}$ for all $j$, there exists a number $N_{j}$ for each $j$ such that $x_{n} \in U_{0 j}$ for all $n>N_{j}$. Let

$$
W_{n}=\bigcap_{\substack{i=0 \\ i \neq n}}^{N_{n}} U_{n i} \cap \bigcap\left\{U_{0 k} \mid 1 \leq k<n, x_{n} \in U_{0 k}\right\} .
$$

Then each $W_{n}$ is open, being the intersection of finitely many open sets. We show that $W_{m} \cap W_{n}=\varnothing$ for all $m$ and $n$ such that $m<n$. Suppose that $n \leq$ $N_{m}$. Then $W_{m} \subset U_{m n}$ and $W_{n} \subset U_{n m}$. Since $U_{m n} \cap U_{n m}=\varnothing, W_{m} \cap W_{n}=\varnothing$. Suppose, to the contrary, that $n>N_{m}$. Then $x_{n} \in U_{0 m}$, so $W_{n} \subset U_{0 m}$. Since $W_{m} \subset U_{m 0}$ and $U_{0 m} \cap U_{m 0}=\varnothing, W_{m} \cap W_{n}=\varnothing$. Thus the sets $W_{n}$ are indeed pairwise disjoint.

Lemma 7. Let $Y$ be first countable and locally path-connected. Then $Y$ has at each point $y_{0}$ a decreasing sequence of path-connected open sets forming a local basis.

Proof. Let $\left\{G_{n}\right\}$ be a countable local basis for $Y$ at $y_{0}$. Let $G_{n}^{1}=\bigcap_{k=1}^{n} G_{k}$ for all $n$. Since $Y$ is locally path-connected, there exist path-connected open neighborhoods $G_{n}^{2}$ of $y_{0}$ for each $n$ such that $G_{n}^{2} \subset G_{n}^{1}$. Let $G_{n}^{3}=\bigcup_{k=n}^{\infty} G_{k}^{2}$ for all $n$. Then $\left\{G_{n}^{3}\right\}$ is also a countable local basis at $y_{0}$ because $G_{k}^{2} \subset G_{k}^{1} \subset G_{n}$ for all $k \geq n$ and so $G_{n}^{3} \subset G_{n}$. We show that the sets $G_{n}^{3}$ are path-connected. Fix two points $x, z \in G_{n}^{3}$. Then there exist numbers $k, m$ such that $x \in G_{k}^{2}$ and $z \in G_{m}^{2}$. Since $y_{0} \in G_{n}^{2}$ for all $n$, there exist paths $p, q: I \rightarrow Y$ such that $p(0)=x, p(1)=q(0)=y_{0}$, and $q(1)=z$. Let

$$
r(x)= \begin{cases}p(2 x), & x \leq \frac{1}{2} \\ q(2 x-1), & x \geq \frac{1}{2} .\end{cases}
$$

Then $r$ is continuous, $r(0)=x$, and $r(1)=z$. Finally, we show that $G_{n+1}^{3} \subset$ $G_{n}^{3}$, i.e., the sequence $G_{n}^{3}$ is decreasing. For all $n$, we have

$$
G_{n}^{3}=\bigcup_{k=n}^{\infty} G_{k}^{2} \supset \bigcup_{k=n+1}^{\infty} G_{k}^{2}=G_{n+1}^{3} \text {. }
$$

Thus $\left\{G_{n}^{3}\right\}$ is the required sequence. 
The first generalization of Luzin's Theorem places fewer conditions on the domain $X$ of the functions $f$ than the second generalization will, and no conditions at all on the range $Z$ of $F$, but only at the expense of supposing that $F$ has continuous $x$-sections. We note that the condition that $X \times Y$ be sequential may be satisfied either by taking $X$ to be first countable (by Lemmas 2 and 3) or by taking $X$ to be sequential and either $X$ or $Y$ to be sequentially compact (by Lemma 4).

Theorem 1. Let $X$ be completely regular. Let $Y$ be first countable and locally path-connected. Let $Z$ be a topological space. Suppose that $X \times Y$ is sequential, that the function $F: X \times Y \rightarrow Z$ has continuous $x$-sections, and that for any continuous function $f: X \rightarrow Y$, the function $F_{f}$ is continuous. Then $F$ is also continuous.

Proof. Suppose that $F$ is discontinuous. Then there is an open set $W \subset Z$ such that $F^{-1}(W)$ is not open in $X \times Y$. Since $X \times Y$ is sequential, there must be a sequence $\left\{\left(x_{n}, y_{n}\right)\right\}$ in $(X \times Y) \backslash F^{-1}(W)$ converging to a point $\left(x_{0}, y_{0}\right)$ in $F^{-1}(W)$. But since $W$ is an open neighborhood of $F\left(x_{0}, y_{0}\right)$ and $F\left(x_{n}, y_{n}\right) \notin W$ for all $n,\left\{F\left(x_{n}, y_{n}\right)\right\}$ cannot even accumulate to $F\left(x_{0}, y_{0}\right)$. Therefore $F\left(x_{0}, y_{0}\right)$ is not a limit point of any subsequence of $\left\{F\left(x_{n}, y_{n}\right)\right\}$.

Let $\left\{\left(x_{n}^{1}, y_{n}^{1}\right)\right\}$ be the sequence of all $\left(x_{n}, y_{n}\right)$ such that $x_{n}=x_{0}$. Suppose that $\left\{\left(x_{n}^{1}, y_{n}^{1}\right)\right\}$ is infinite. Then since $F$ has continuous $x$-sections, by Lemma 1 we have $\lim F\left(x_{n}^{1}, y_{n}^{1}\right)=\lim F\left(x_{0}, y_{n}^{1}\right) \ni F\left(x_{0}, y_{0}\right)$. This contradicts our earlier conclusion that $F\left(x_{0}, y_{0}\right)$ is not a limit point of any subsequence of $\left\{F\left(x_{n}, y_{n}\right)\right\}$. Thus $\left\{\left(x_{n}^{1}, y_{n}^{1}\right)\right\}$ is finite. Let $\left\{\left(x_{n}^{2}, y_{n}^{2}\right)\right\}$ be the sequence of all $\left(x_{n}, y_{n}\right)$ such that $x_{n} \neq x_{0}$. Then $\left\{\left(x_{n}^{2}, y_{n}^{2}\right)\right\}=\left\{\left(x_{n}, y_{n}\right)\right\} \backslash\left\{\left(x_{n}^{1}, y_{n}^{1}\right)\right\}$ is infinite, so by Lemma 1 it converges to $\left(x_{0}, y_{0}\right)$. Then, by Lemma $5,\left\{x_{n}^{2}\right\}$ has a subsequence of distinct points $\left\{x_{n}^{3}\right\}$. For each $n$ choose $m$ such that $x_{n}^{3}=x_{m}^{2}$ and let $y_{n}^{3}=y_{m}^{2}$. Then $\left\{\left(x_{n}^{3}, y_{n}^{3}\right)\right\}$ is a subsequence of $\left\{\left(x_{n}^{2}, y_{n}^{2}\right)\right\}$ with distinct $x$-coordinates.

Let $\left\{G_{n}\right\}$ be a decreasing sequence of path-connected open sets forming a basis for $Y$ at $y_{0}$ as in Lemma 7. Since $\left\{y_{n}^{3}\right\}$ converges to $y_{0}$ and $G_{1}$ is an open neighborhood of $y_{0}$, there exists a number $N$ such that $y_{n}^{3} \in G_{1}$ for all $n>N$. Let $\left(x_{n}^{4}, y_{n}^{4}\right)=\left(x_{n+N}^{3}, y_{n+N}^{3}\right)$ for all $n \geq 1$. Then $y_{n}^{4} \in G_{1}$ for all $n$, so that $m_{n}=\sup \left\{m \mid 1 \leq m \leq n, y_{n}^{4} \in G_{m}\right\}$ exists for each $n$. Since $G_{m_{n}}$ is path-connected for all $n$, there exist paths $p_{n}: I \rightarrow Y$ such that $p_{n}(0)=y_{0}$, $p_{n}(1)=y_{n}^{4}$, and $p_{n}(I) \subset G_{m_{n}}$ for all $n$. Since $\left\{x_{n}^{4}\right\} \subset\left\{x_{n}^{3}\right\},\left\{x_{n}^{4}\right\}$ is a sequence of distinct points and $x_{n}^{4} \neq x_{0}$ for all $n$. Therefore, by Lemma 6 , there exist pairwise disjoint open neighborhoods $W_{n}$ of $x_{n}^{4}$ for each $n$. Furthermore, since $X$ is completely regular, there exist continuous functions $f_{n}: X \rightarrow I$ such that $f_{n}\left(x_{n}^{4}\right)=1$ and $f_{n}\left(X \backslash W_{n}\right)=0$ for all $n$.

Let

$$
f(x)= \begin{cases}p_{n}\left(f_{n}(x)\right), & x \in W_{n}, \\ y_{0}, & x \notin \bigcup_{n=1}^{\infty} W_{n} .\end{cases}
$$

Let $V$ be open in $Y$. If $y_{0} \notin V$, then $f^{-1}(V)=\bigcup_{n=1}^{\infty} f_{n}^{-1}\left(p_{n}^{-1}(V)\right)$ is open. If $y_{0} \in V$, then there exists a number $M$ such that $G_{M} \subset V$. Since $\left\{y_{n}^{4}\right\}$ converges to $y_{0}$ and $G_{M}$ is an open neighborhood of $y_{0}$, there exists a number $N$ such that $y_{n}^{4} \in G_{M}$ for all $n>N$. Therefore $p_{n}(I) \subset G_{m_{n}} \subset G_{M} \subset V$ for 
each $n>\max (M, N)$, where $m_{n}$ was defined above for each $n$. Therefore

$$
f^{-1}(V)=X \backslash f^{-1}(Y \backslash V)=X \backslash \bigcup_{n=1}^{N} f_{n}^{-1}\left(p_{n}^{-1}(Y \backslash V)\right)
$$

is open, being the complement of the union of finitely many closed sets. Thus $f$ is continuous, so

$$
\lim F\left(x_{n}^{4}, y_{n}^{4}\right)=\lim F\left(x_{n}^{4}, f\left(x_{n}^{4}\right)\right)=\lim F_{f}\left(x_{n}^{4}\right) \ni F_{f}\left(x_{0}\right)=F\left(x_{0}, y_{0}\right) .
$$

This contradicts our assumption that $F$ is discontinuous, from which we deduced that $F\left(x_{0}, y_{0}\right)$ is not a limit point of any subsequence of $F\left(x_{n}, y_{n}\right)$.

The second generalization of Luzin's Theorem dispenses with the condition that $F$ have continuous $x$-sections by assuming additional hypotheses on the spaces $X$ and $Z$, including the assumption that $X$ is dense in itself. Note that one must assume something like this about $X$ if $F$ need not have continuous $x$-sections because of the trivial example $X=\{0,1\}, Y=Z=[0,1]$, $F(x, 0)=0$, and $F(x, y)=1$, otherwise. $F$ is discontinuous, but $F_{f}$ is continuous for every continuous $f: X \rightarrow Y$. Since much of the proof of Theorem 2 is identical to the proof of Theorem 1 , we give only the additions to that proof needed to prove this theorem.

Theorem 2. Let $X$ be first countable, dense in itself, and completely regular. Let $Y$ be first countable and locally path-connected. Let $Z$ be first countable and $T_{3}$. Given a function $F: X \times Y \rightarrow Z$, suppose that for every continuous function $f: X \rightarrow Y$ the function $F_{f}$ is continuous. Then $F$ is continuous.

Proof. The alterations to the proof of Theorem 1 required to prove Theorem 2 follow. First, since $X$ and $Y$ are first countable, $X \times Y$ is first countable by Lemma 2 , hence sequential by Lemma 3 . Next, if the sequence $\left\{\left(x_{n}^{1}, y_{n}^{1}\right)\right\}$ is infinite, we argue as follows:

Let $\left\{U_{n}\right\}$ and $\left\{V_{n}\right\}$ be countable bases for $X$ at $x_{0}$ and for $Z$ at $F\left(x_{0}, y_{0}\right)$, respectively. For any open neighborhood $J$ of $F\left(x_{0}, y_{0}\right), Z \backslash J$ is a closed set not including $F\left(x_{0}, y_{0}\right)$. Since $Z$ is $T_{3}$, there exist disjoint open neighborhoods $U$ and $V$ of $F\left(x_{0}, y_{0}\right)$ and $Z \backslash J$, respectively. Since $\left\{V_{n}\right\}$ is a countable basis at $F\left(x_{0}, y_{0}\right)$, there exists a number $m$ such that $V_{m} \subset U$. Then, since $U \subset(Z \backslash V) \subset J$ and $Z \backslash V$ is closed, the closure of $V_{m}$ is a subset of $Z \backslash V$, which in turn is a subset of $J$. Let $K_{n}$ be the closure of $V_{n}$ for each $n$. Then for any open neighborhood $J$ of $F\left(x_{0}, y_{0}\right)$, there exists $n$ such that $K_{n} \subset J$.

Let $g_{n}: X \rightarrow Y$ be defined by $g_{n}(x)=y_{n}$ for each $n$. Then let

$$
J_{n}=\bigcap\left\{U_{i} \mid 1 \leq i \leq n, x_{n} \in U_{i}\right\} \backslash F_{g_{n}}^{-1}\left(\bigcup\left\{K_{j} \mid 1 \leq j \leq n, F\left(x_{n}, y_{n}\right) \notin K_{j}\right\}\right) \text {, }
$$

where we make the convention that the intersection of the empty family of subsets of $X$ is $X$ itself. Note that $x_{n} \in J_{n}$ and $J_{n}$ is open for each $n$, being the difference of a finite intersection of open sets and the continuous inverse image of a finite union of closed sets. Since $X$ is dense in itself, this means that each $J_{n}$ includes at least two points, and in particular, $J_{n} \backslash\left\{x_{0}\right\}$ is nonempty. Now for all $n$, choose $x_{n}^{2} \in J_{n} \backslash\left\{x_{0}\right\}$ and let $y_{n}^{2}=y_{n}$. We show that the sequence $\left\{x_{n}^{2}\right\}$ converges to $x_{0}$. Let $U$ be an open neighborhood of $x_{0}$. Then 
there exists $m$ such that $U_{m} \subset U$. Since $U_{m}$ is an open neighborhood of $x_{0}$ and $\left\{x_{n}\right\}$ converges to $x_{0}$, there exists $N$ such that $x_{n} \in U_{m}$ for all $n>N$. Then $x_{n}^{2} \in U_{m} \subset U$ for all $n>\max (m, N)$ by the choice of $x_{n}^{2}$. Therefore $\left\{x_{n}^{2}\right\}$ converges to $x_{0}$, as desired.

Finally, after having shown that a subsequence of $\left\{F\left(x_{n}^{2}, y_{n}^{2}\right)\right\}$ converges to $F\left(x_{0}, y_{0}\right)$, as was done in the proof of Theorem 1 , we show that the corresponding subsequence of $\left\{F\left(x_{n}, y_{n}\right)\right\}$ converges to $F\left(x_{0}, y_{0}\right)$. Let $V$ be an open neighborhood of $F\left(x_{0}, y_{0}\right)$. Then there exists a number $m$ such that $K_{m} \subset V$. If $\left\{F\left(x_{n}^{2}, y_{n}^{2}\right)\right\}$ converges to $F\left(x_{0}, y_{0}\right)$, then there exists a number $N$ such that $F\left(x_{n}^{2}, y_{n}^{2}\right) \subset V_{m} \subset K_{m}$ for all $n>N$. Then $F\left(x_{n}, y_{n}\right) \in K_{m} \subset V$ for all $n>\max (m, N)$, by the choice of $x_{n}^{2}$. Therefore $\left\{F\left(x_{n}, y_{n}\right)\right\}$ converges to $F\left(x_{0}, y_{0}\right)$.

We now present two counterexamples to show the necessity for some of the hypotheses in our theorems. The first counterexample shows that the hypothesis that $Y$ is locally path-connected cannot be omitted from either theorem. The space $Y$ given in the example is in fact not locally connected, so this example does not rule out the possibility that local connectedness could replace local path-connectedness in the hypotheses of either theorem.

Example 1. The Perforated Unit Interval. Let $Y=I \backslash\left\{1, \frac{1}{2}, \frac{1}{3}, \ldots\right\}$. Let $X=Z=I$. Then if $f: X \rightarrow Y$ is continuous, $f(X)$ is connected (and locally connected) since $X$ is. If $0 \in f(X)$, then $f(X)=\{0\}$. Otherwise, let $y \in f(X) \backslash\{0\}$. Then there exists a number $n$ such that $1 / n<y$, and $f^{-1}([0,1 / n))$ and $f^{-1}((1 / n, 1])$ are disjoint nonempty open sets whose union is $X$, contradicting the connectedness of $I$. Let

$$
F(x, y)= \begin{cases}0, & (x, y)=(0,0) \\ x y /\left(x^{2}+y^{2}\right), & (x, y) \neq(0,0) .\end{cases}
$$

Then $F$ is continuous except at $(0,0)$, and $F$ is separately continuous everywhere. Since the only continuous function through $(0,0)$ is constant, $F$ satisfies the hypotheses of both theorems, but is not continuous.

The second counterexample uses a space that was first described by Hewitt in [2], or rather a variant of this space that is described in [4]. This counterexample shows that $F$ need not be continuous if all the hypotheses of Theorem 1 are satisfied except the complete regularity of $X$ and possibly the sequentiality of $X \times Y{ }^{1}$

Example 2 [4, pp. 111-113]. Hewitt's Condensed Corkscrew. We refer the interested reader to [4] for the construction and properties of this space, which we call $X$ here. We will use the facts that $X$ is regular, but not completely regular, and that every continuous real-valued function on $X$ is constant. We also need a nonrepeating convergent sequence in $X$. In the notation and terminology of [4], the sequence $\{(0, n)\}$ in any plank of any of the "Tychonoff Corkscrews" of $X$ converges to the point $(0, \omega)$ in the same plank in the product topology on $A$, hence in the weaker condensed topology on $A$. Since this sequence and its limit point are included in $X$, the sequence converges in $X$.

\footnotetext{
${ }^{1}$ As far as I know, the sequentiality of Hewitt's “Corkscrew" has not been determined [4, p. 198].
} 
Now let $Y=I$ and let $Z$ be Sierpiński space [4, pp. 44-46]. Let $\left\{x_{n}\right\}$ be a nonrepeating convergent sequence in $X$ that does not include its limit. Define $F\left(x_{n}, 1 / n\right)=1$ and $F(x, y)=0$ for all other points $(x, y)$ of $X \times Y$. Then $F$ is discontinuous since $\lim F\left(x_{n}, 1 / n\right)=\lim 1=1$ whereas $F\left(\lim \left(x_{n}, 1 / n\right)\right)=$ $F\left(\lim \left(x_{n}\right), 0\right)=0$. Nevertheless, we will show that $F$ has continuous $x$ sections and is continuous on the graph of every continuous function from $X$ to $Y$. Since $\{1\}$ is the only nontrivial closed set in Sierpinski space, a function $f$ into Sierpiński space is continuous if and only if $f^{-1}(1)$ is closed. For any $x \in X$, let $F_{x}$ be the restriction of $F$ to $\{x\} \times Y$. Then $F_{x_{n}}^{-1}(1)=\left\{\left(x_{n}, 1 / n\right)\right\}$. Since $X$ and $Y$ are both $T_{1}$, their product $X \times Y$ is $T_{1}$ also and so all singletons in $X \times Y$ are closed, in particular the singleton sets $\left\{\left(x_{n}, 1 / n\right)\right\}$. For other values of $x, F_{x}^{-1}(1)=\varnothing$, which is closed in any space. Thus $F$ has continuous $x$-sections. Now let $f$ be a continuous (hence constant) function from $X$ to $Y$. Then $F_{f}^{-1}(1)=x_{n}$ if $f$ is identically $1 / n$, or $F_{f}^{-1}(1)=\varnothing$ otherwise. Thus $F_{f}$ is continuous for every continuous $f$.

\section{REFERENCES}

1. R. Engelking, General topology, PWN-Polish Scientific Publishers, Warsaw, 1977.

2. E. Hewitt, On two problems of urysohn, Ann. of Math. (2) 47 (1946), 503-509.

3. N. N. Luzin, Teorilâ funktsìi deistvitel'nogo peremennogo, 2nd ed., National Educational Publishers, Moscow, 1948.

4. L. A. Steen and J. A. Seebach, Counterexamples in topology, 2nd ed., Springer-Verlag, New York, 1978.

Department of Mathematics, Cornell University, Ithaca, New York 14853

E-mail address: dalbec@math.cornell.edu 